\title{
Acoustic Emissions of Semi-Permeable Trailing Edge Serrations
}

\author{
Carlos Arce León ${ }^{1,2}$ (1) • Roberto Merino-Martínez ${ }^{2}$ - Stefan Pröbsting ${ }^{2} \cdot$ Daniele Ragni $^{2}$ • \\ Francesco Avallone ${ }^{2}$
}

Received: 30 January 2017 / Accepted: 9 May 2017 / Published online: 22 May 2017

(C) The Author(s) 2017. This article is an open access publication

\begin{abstract}
The trailing edge of a NACA 0018 airfoil is modified through the attachment of serrations with different degrees of permeability. Acoustic beamforming is used to inspect the turbulent boundary layer-trailing edge noise emissions from the unmodified and serrated trailing edges. Different freestream velocities and angles of attack are investigated. The serration permeability is prescribed by having slits cut into the solid surface of the serrations in two different configurations. The results indicate that a certain benefit in noise reduction is obtained from a mixed solid/slitted configuration, while a fully slitted configuration loses most of the noise reduction performance.
\end{abstract}

Keywords Aeroacoustics · Trailing edge serrations - Acoustic beamforming

\section{Introduction}

Over the last years, trailing edge serrations have become the predominant device for noise reduction in industrial wind turbine blade applications $[17,21,24]$. Their effectiveness has been well established in wind tunnel experiments $[6,12,15$, 22], with measured noise reduction levels up to $8 \mathrm{~dB}$. The Reynolds numbers there investigated go roughly from $0.2 \times$ $10^{6}$ to $1 \times 10^{6}$. In [15], for example, single microphones were used to characterize the noise directivity. A wide frequency range was presented, from around 20 to $20 \times 10^{4} \mathrm{~Hz}$, while the range where noise reduction was observed was found to be case dependent, typically ranging from around $200 \mathrm{~Hz}$ to a Strouhal number-dependent upper limit [15].

Despite the research available, there is still debate about the physical mechanisms. Two mechanisms have been hypothesized. The first argues that serrations beneficially

Carlos Arce León

caar@1mwindpower.com

1 Aerodynamics and Acoustics Group, LM Wind Power R\&D, J. Duikerweg 15-A, 1703 DH Heerhugowaard, The Netherlands

2 Faculty of Aerospace Engineering, Delft University of Technology, Kluyverweg 1, 2629 HS Delft, The Netherlands modify the flow [11,19], and a second argues that the most contributing factor is the reduction of the scattering efficiency by the modification of the trailing edge geometry $[13,16]$.

Traditionally, the most common design has consisted of thin, triangular solid panels. Designs which incorporate surface permeability were investigated in [14]. This was achieved by the introduction of holes or slits in the material. More recently, a variation of this design has been proposed by Oerlemans [24].

The solid serrations have been found to reduce noise, but are known to increase it beyond a certain frequency $[6,15]$. This unwanted effect can lead to the degradation of the overall noise reduction performance in industrial applications or limit the application of serrations to a range of conditions. Yet this seems to be mostly averted by the slitted serrations tested in [14], showing a promising direction toward a new design alternative. An attempt to replicate this result is thus presented here. In addition, the noise reduction performance of a new hybrid design is explored.

Two variations of the slitted serration designs and two solid serrations with different lengths (Fig. 1) are compared, using the unmodified straight trailing edge as the baseline reference. The geometry of the serration labeled Slitted has been chosen based on the best-performing serration in [14]. 
Fig. 1 Serration model

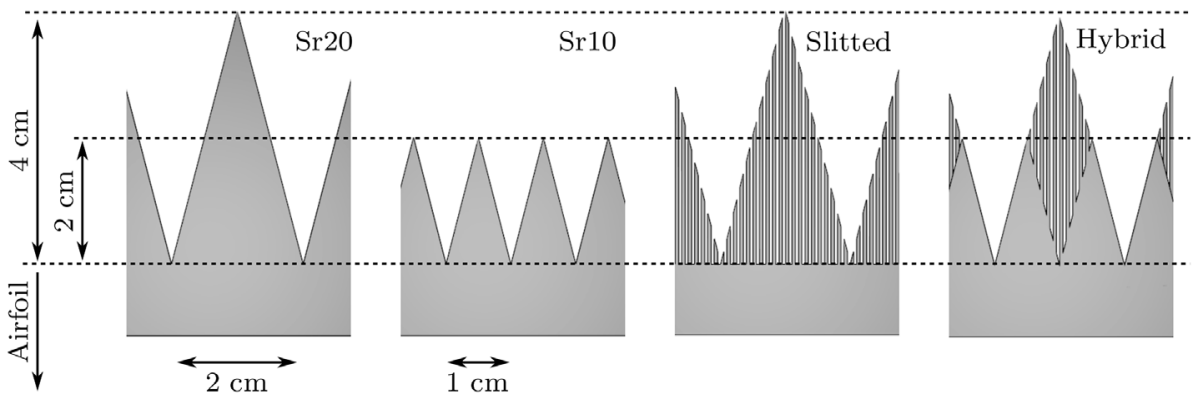
schematic

The serrations have been mounted on a NACA 0018 airfoil. This symmetric airfoil has been preferred, as equal flow conditions are obtained on the upper and lower sides at $\alpha=0^{\circ}$. This differs from [14], where a non-symmetric NACA 6512-10 airfoil was used. Three freestream velocities were investigated, 30,35 and $40 \mathrm{~m} / \mathrm{s}$, with the highest corresponding to a Reynolds number of around 526,000, and two angles of attack, $\alpha=0^{\circ}$ and $\alpha=12^{\circ}$ (the geometric angle of attack is indicated).

The TBL-TE noise was measured by means of acoustic beamforming using a microphone array. This method was preferred in order to obtain the acoustic signals from sound sources on the trailing edge location and avoid extraneous noise sources in the background [5]. In comparison, [14] used single microphones to obtain the noise levels.

The current study compares the acoustic emissions of the serrated edge to the unmodified straight edge of the airfoil. This is done in view of the state-of-the-art application of serrations in the wind turbine industry [21,24]. For this purpose, the objective of serrations is to mitigate the noise of state-of-the-art airfoil designs without prior trailing edge modification.

\section{Experimental Setup}

The experiments were conducted at the Delft University of Technology Vertical Wind Tunnel (V-Tunnel). It is a lowturbulence ( $\lesssim 1 \%$ turbulence intensity) wind tunnel. An open test section was used, allowing a direct line of sight to the relevant noise sources from the trailing edge (Fig. 2).

A $C=20 \mathrm{~cm}$ chord length NACA 0018 airfoil was used. To ensure that the noise source observed was TBLTE noise, the boundary layer was tripped at $0.2 \cdot C$ using three-dimensional roughness elements following the recommendations in [10]. It was confirmed to remain turbulent up to the trailing edge using a microphone probe.

The serrations were inserted in the trailing edge using a modular system, as shown in Fig. 3. The straight edge refers to the unmodified trailing edge of the airfoil.

The length of the solid serrations is established as a ratio with the airfoil chord length. The serrations that were $0.2 \cdot C=4 \mathrm{~cm}$ in length are labeled $\mathrm{Sr} 20$, and the $0.1 \cdot C=2$ $\mathrm{cm}$ long serrations are labeled Sr10. The semi-permeable designs, labeled slitted and hybrid, along with the solid serrations, can be observed in Fig. 1. The solid area of the hybrid design was the same as Sr10, while the length of the semi-
Fig. 2 Wind tunnel setup (left) indicating the microphone array location with respect to the airfoil and microphone configuration on the array (right)
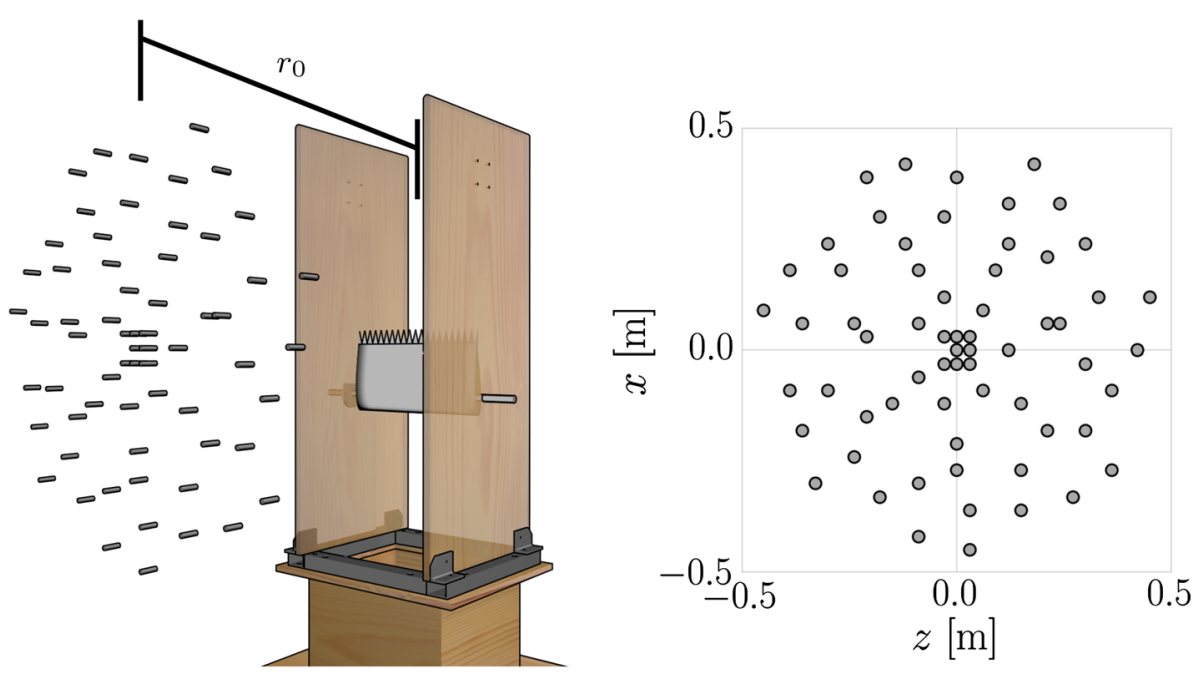


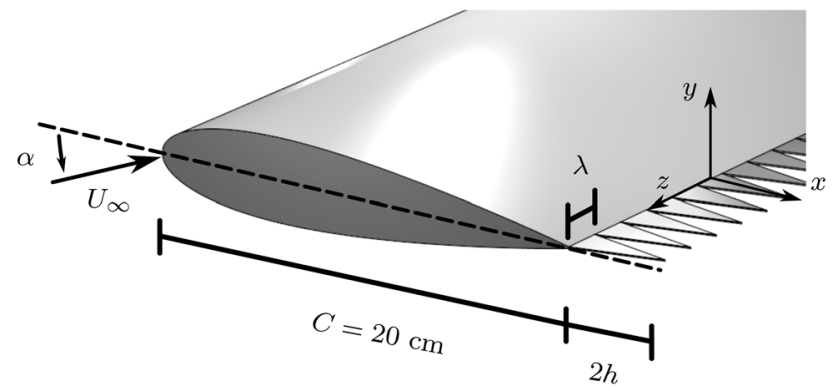

Fig. 3 Airfoil and serration schematic indicating the coordinate system

permeable area in both designs was the same as for $\operatorname{Sr} 20$. The serration root of all the designs aligns with the original trailing edge of the airfoil.

The serration length was chosen following the design guidelines presented in [15], where it is recommended to be at least twice the boundary layer thickness at the straight trailing edge location, $\delta$. For the current setup, $\delta$ was measured using particle image velocimetry (PIV) [5], and the serration length was confirmed to satisfy this recommendation. At $\alpha=0^{\circ}$, at the straight trailing edge location, $\delta=9.4 \mathrm{~mm}$.

The design of the slitted serrations followed that of [14]. $0.5 \mathrm{~mm}$ cutouts were made in the material, leaving $0.5 \mathrm{~mm}$ slits. The cutouts end at the base of the serration. The same slit dimensions were used in the hybrid design.

Figure 2 shows a schematic of the experiment setup, along with the microphone array and its location with respect to the test section. The distribution of the 64-microphone array is also shown. It follows a multi-arm logarithmic spiral configuration $[23,26]$ with a diameter of $0.9 \mathrm{~m}$.

The center microphone of the array was aligned with the middle point of the trailing edge (for the case with $\alpha=0^{\circ}$ ). The distance from the array plane to the airfoil trailing edge ( $r_{0}$ in Fig. 2) varied between 1.05 and $1.26 \mathrm{~m}$. The beamforming method accounts for the distance, and a reference distance of $1 \mathrm{~m}$ was used to present the results in the remainder of this paper. To further avoid unwanted sources of noise, the airfoil leading edge was located $0.5 \mathrm{~m}$ from the wind tunnel nozzle and the side-plate edges.

The spatial resolution $\Delta R$ (i.e., the minimum distance at which two different sound sources can be separated) of the phased microphone array can be estimated using Rayleigh's criterion with the following equation

$\Delta R \sim Y \tan \left(1.22 \frac{c}{f D}\right)$

where $Y$ is the distance from the array plane to the scan plane, $c$ is the sound speed, $f$ is the sound frequency, and $D$ is the array diameter. This parameter limits the lowest frequency that can be analyzed. In this experiment, a frequency of $800 \mathrm{~Hz}$ was considered as a lower limit to avoid the influence of extraneous noise sources coming from the wind tunnel system.

A sampling frequency of $50 \mathrm{kHz}$ and a recording time per measurement of $60 \mathrm{~s}$ were employed. To calculate the ensemble average of the cross-spectral matrix, the acoustic data were averaged using time blocks of 2048 samples ( $\Delta t=40.96 \mathrm{~ms}$ ) for each Fourier transform and a Hanning window with $50 \%$ data overlap. With these parameter values, the frequency resolution is $24.41 \mathrm{~Hz}$ and the expected error [9] in the estimate for the cross-spectrum was $2.6 \%$.

For beamforming, a scan grid covering the expected area of noise generation was selected, ranging from $z=-0.22 \mathrm{~m}$ to $z=0.22 \mathrm{~m}$ in the spanwise direction and from $x=-0.3 \mathrm{~m}$ to $x=0.2 \mathrm{~m}$ in the streamwise direction, according to the axes defined in Fig. 2, with a distance between grid points of $1 \mathrm{~mm}$. Thus, the resulting grid size is $441 \times 501$.

Each microphone was previously calibrated using a pistonphone, and the array was tested by using tonal sound generated with a speaker at a known position emitting at several single frequencies. The signal-to-noise ratio (SNR) of this setup was found to be between 10 and $24 \mathrm{~dB}$ for the frequency range of interest $1-5 \mathrm{kHz}$ for all cases.

The shear layer effect in the acoustic measurements [1] was considered negligible because of the small angle $\left(<10^{\circ}\right)$ between the center of the array and the scan area of interest and the considerably low freestream velocities employed in this experiment [27].

\section{Measurement Methodology}

For this paper, delay-and-sum beamforming, also known as conventional frequency domain beamforming [18,29], was applied to the acoustic data, due to its simplicity, robustness and low computational cost.

Since TBL-TE noise is assumed to be a distributed sound source, the source maps were integrated over an area extending from $z=-0.1 \mathrm{~m}$ to $z=0.1 \mathrm{~m}$ and from $x=-0.06 \mathrm{~m}$ to $x=0.06 \mathrm{~m}$ (see Fig. 4). This region was selected to reduce the contribution from extraneous sound sources, while still containing a representative part of the trailing edge noise [25].

The beamforming results in that region were further normalized by the value of the integral of a simulated line source of monopoles of unitary strength placed horizontally at the trailing edge position $(x=0 \mathrm{~m}$ and from $z=-0.1 \mathrm{~m}$ to $z=0.1 \mathrm{~m}$ ), evaluated within the same area. This method was proposed by Sijtsma [30], and it is a similar approach as the covariance matrix fitting algorithm $[31,32]$ applied to the source power integration technique [29]. This method was proven to provide very accurate results in the array methods benchmark [28] for a simulated line source heavily contaminated with background noise. In addition, for this paper, the 


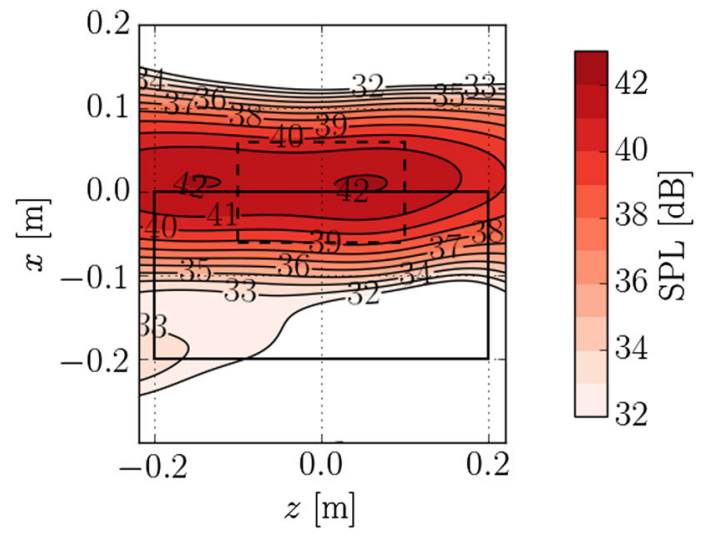

Fig. 4 One-third octave band $(3150 \mathrm{~Hz})$ beamforming source plot for the straight edge airfoil with $U_{\infty}=40 \mathrm{~m} / \mathrm{s}$ and $\alpha=0^{\circ}$. The solid black line represents the airfoil position, and the dashed black line the integration region

main diagonal of the cross-spectral matrix was deleted to reduce the influence of incoherent background noise.

This integration process was then repeated for each frequency of interest to obtain the acoustic frequency spectra of the trailing edge for the different serrations configurations.

\section{Results}

The integrated sound pressure level (SPL) at a reference distance of $1 \mathrm{~m}$ of the four serrated geometries and the straight edge is observed in Fig. 5. The three investigated freestream velocities are shown for $\alpha=0^{\circ}$. Additionally, the background noise is added as reference. The noise reduction by all four serrations is evident.

To better illustrate the levels of noise reduction obtained, the same data are presented relative to the straight edge in Fig. 6. A positive $\triangle$ SPL represents a reduction in noise.

Reduction levels of up to $8 \mathrm{~dB}$ are achieved by the hybrid serrations. Serrations Sr20 perform similar, exhibiting only slightly lower reduction levels. The short serrations, Sr10, perform about $2 \mathrm{~dB}$ worse across the observed spectrum. The slitted design proves to be most inefficient, especially at frequencies below $2.5 \mathrm{kHz}$. This result disagrees with what has been observed in [14], where this design performed similar or better than the equivalent solid serration design. It should be noted that the airfoil used in [14] was not symmetric.
Fig. 5 Absolute integrated SPL for the four serrated geometries and the straight edge at $\alpha=0^{\circ}$

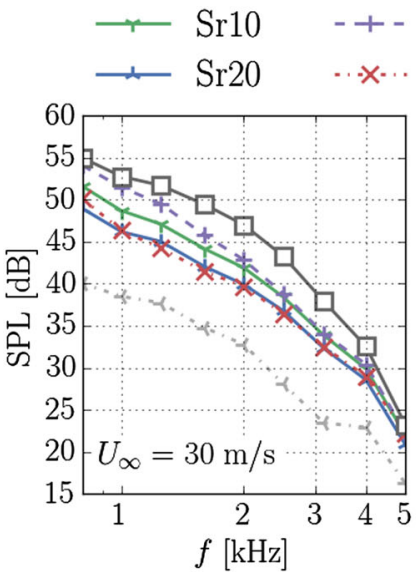

Slitted $\quad \square-$ Straight Hybrid $\quad \cdots \nrightarrow \cdots$ Background
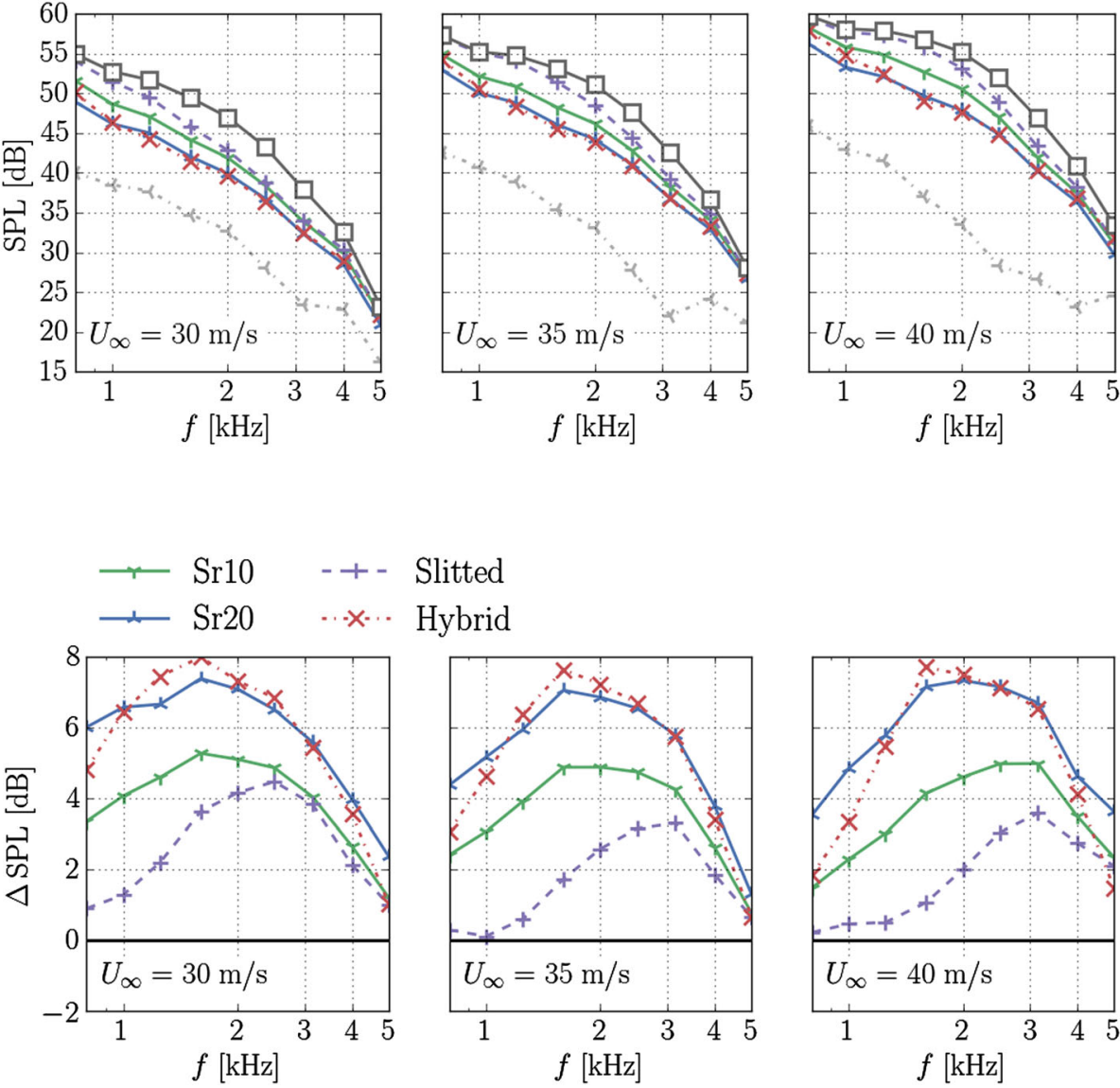

Slitted Hybrid
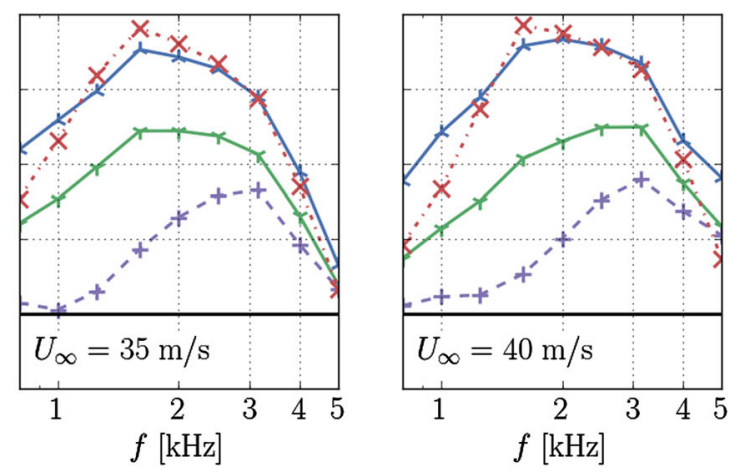

Fig. 6 Relative integrated SPL for the four serrated geometries with respect to the straight edge at $\alpha=0^{\circ}$ 
Fig. 7 Relative integrated SPL for the four serrated geometries with respect to the straight edge at $\alpha=12^{\circ}$

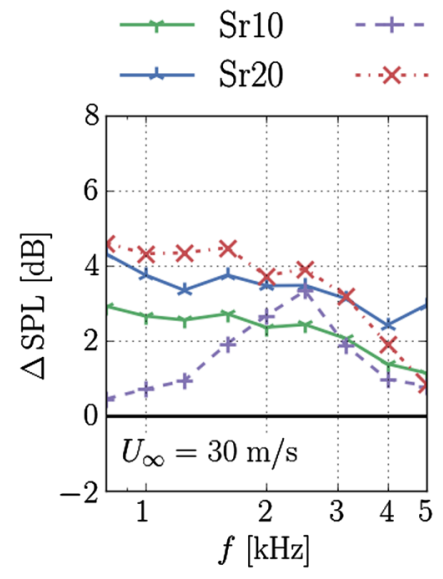

Slitted

Hybrid

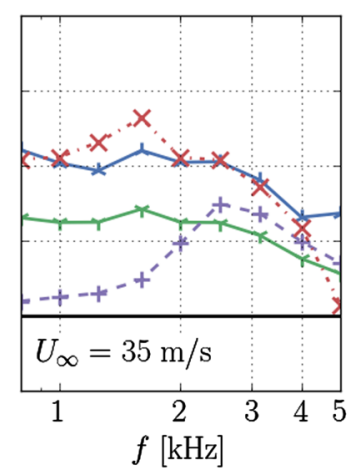

The reduction level trends vary moderately across the investigated velocities, showing some slight degradation at lower frequencies.

The results at $\alpha=12^{\circ}$, shown in Fig. 7, exhibit a weakened noise reduction performance for all the serration designs. Sr20 and hybrid remain as the designs that reduce most noise, topping almost at $6 \mathrm{~dB}$. Negligible trend differences are again observed with increasing velocity, suggesting that the noise reduction performance is only weakly affected by it.

\section{Discussion}

All investigated serration designs provided a reduction of the noise compared to the straight edge. Differences between them were nevertheless observed.

In the current experiment, the serrations were investigated at a zero flow incidence, in the $\alpha=0^{\circ}$ case. This was achieved by using a symmetric airfoil and no serration flap angle. The same flow conditions were thereby attained over the upper and lower serration sides.

Under these conditions, only small or negligible differences have been found in the mean boundary layer features over the Sr20 and the slitted serrations [3]. It is suggested therefore that the poor noise reduction achieved with the slitted serrations, compared to Sr20, is likely explained from a standpoint of the scattering efficiency reduction by the geometrically modified edge.

An argued intent of the serrations is to avert a trailing edge design which is perpendicular to the flow direction, thereby alleviating the impedance discontinuity at the edge $[8,16,20]$. The slitted serration design nevertheless retains a strong impedance change at the location where the slits originate, where it preserves $50 \%$ of the wetted edge of the straight trailing edge. It has been furthermore shown that at this location the intensity of the acoustic source term is larger [7], possibly having a more critical effect on noise. It is therefore likely that this design feature fails to achieve the intended relaxation of the impedance discontinuity. The hybrid serrations instead successfully recover the alleviation of that discontinuity, even outperforming $\mathrm{Sr} 20$ in noise reduction at around $1.8 \mathrm{kHz}$.

The performance of the hybrid design suggests that the slits are indeed not an unwanted feature, but they need to be carefully integrated into a proper design. It is further interesting to note that, despite that the hybrid design has the same solid serration contour as $\mathrm{Sr} 10$ (see Fig. 1), it consistently reduces more noise than the former, especially in the central frequency range that has been observed.

At $\alpha=12^{\circ}$ the relative noise reduction trends of the different designs remain similar, with the $\mathrm{Sr} 20$ and hybrid serrations being most effective, although by a lesser degree.

It is worth noting that the mean flow in the boundary layer is known to be heavily modified by the solid serration when serration-flow misalignment is prescribed $[2,6,7]$. Strong streamwise vortical structures have been observed originating from the serration edges, and increased turbulence activity has been measured at the pressure side near them [4]. The turbulent structures near the edges are therefore significantly modified and are vastly different on the upper and lower serration sides. Despite this, noise reduction is still observed by up to $4 \mathrm{~dB}$, reinforcing the hypothesis that a crucial factor to achieve noise reduction remains the alleviation of the impedance discontinuity, which is still obtained despite the introduction of serration-flow misalignment and important modifications to the flow.

Slitted serrations, on the other hand, have been shown to exhibit less large-scale mean flow modification [3], likely due to its permeability, allowing an alleviation of the pressure difference between the upper and lower sides of the serrations. Avoiding the observed flow modification of the solid serrations does not appear therefore to be an advantage for improving noise reduction, without an effective relief of the impedance discontinuity. 
Instead, the hybrid serrations, which already appeared to have a more efficient modification of this discontinuity in the $\alpha=0^{\circ}$ case, recover again the levels of noise reduction of $\mathrm{Sr} 20$. Improvement is observed between around 1 and $2 \mathrm{kHz}$, while the effect appears to lose effectiveness for frequencies close to $5 \mathrm{kHz}$. A detailed flow study may reveal the reason for this, but remains otherwise unexplained at this point.

The difference between the noise reduction observed here for the slitted serrations and that observed in [14] can be related to the difference between the investigated airfoil and the material used for manufacturing the serrations. The use of a significantly cambered airfoil in [14] is an important departure from the research conducted here, where equivalent flow conditions over the upper and lower serration sides are enforced by the use of a symmetric airfoil at $\alpha=0^{\circ}$. Furthermore, while the dimensions of the serration and the slits were comparable, the material was different. In the present case, a 1-mm-thick steel sheet was used, while [14] reportedly uses stiff cardboard. Ultimately, while some detailed flow measurements of the slitted serrations used here are available in [3], no similar information is found regarding the serrations of [14]. As a consequence, it remains difficult to formulate an explanation to why such differences were found between the two experiments without having more information about the disparities in the boundary layer flow and general flow topology.

\section{Conclusions}

The noise reduction of several serration designs has been investigated with respect to the unmodified trailing edge of a NACA 0018 airfoil. Several flow velocities and angles of attack were prescribed, and a reduction in the noise was confirmed for all the serrations.

Differences in the reduction levels were nevertheless observed. The slitted serrations exhibit the lowest reduction, while the hybrid and $\mathrm{Sr} 20$ design the highest.

The observations imply that the design of serrations should focus on the alleviation of the impedance discontinuity at the trailing edge, which is suggested to be the primary mechanism to obtain noise reduction. Further benefits may be obtained from beneficially modifying the flow or pressure properties near the edge.

Designs such as the hybrid serrations might be an interesting alternative to the traditional serration design. The significant reduction of TBL-TE noise is supplemented by a smaller wetted surface, compared to solid serrations with a similar noise reduction ( $\mathrm{Sr} 20$, in this case). This is likely to have a lower impact to the aerodynamic performance of the airfoil on which the serrations are installed, a desirable feature for their use in some industrial wind turbine applications.

Open Access This article is distributed under the terms of the Creative Commons Attribution 4.0 International License (http://creativecomm ons.org/licenses/by/4.0/), which permits unrestricted use, distribution, and reproduction in any medium, provided you give appropriate credit to the original author(s) and the source, provide a link to the Creative Commons license, and indicate if changes were made.

\section{References}

1. Amiet, R.K.: Correction of open jet wind tunnel measurements for shear layer refraction. In: 2nd AIAA Aeroacoustics Conference, March 24-26, Hampton, VA, USA (1975)

2. Arce, C., Ragni, D., Pröbsting, S., Scarano, F.: Flow field around a serrated trailing edge at incidence. In: 33rd Wind Energy Symposium. American Institute of Aeronautics and Astronautics, Kissimmee, Florida (2015)

3. Arce León, C., Avallone, F., Pröbsting, S., Ragni, D.: PIV investigation of the flow past solid and slitted sawtooth serrated trailing edges. In: 54th AIAA Aerospace Sciences Meeting. number January, pp. 1-15. American Institute of Aeronautics and Astronautics, San Diego, California (2016)

4. Arce León, C., Merino-Martínez, R., Ragni, D., Avallone, F., Scarano, F., Pröbsting, S., Snellen, M., Simons, D.G., Madsen, J.: Effect of trailing edge serration-flow misalignment on airfoil noise emissions. J. Sound Vib. (2017) (In review)

5. Arce León, C., Merino-Martínez, R., Ragni, D., Avallone, F., Snellen, M.: Boundary layer characterization and acoustic measurements of flow-aligned trailing edge serrations. Exp. Fluids 57(12), 182 (2016)

6. Arce León, C., Ragni, D., Pröbsting, S., Scarano, F., Madsen, J.: Flow topology and acoustic emissions of trailing edge serrations at incidence. Exp. Fluids 57(5), 91 (2016)

7. Avallone, F., Pröbsting, S., Ragni, D.: Three-dimensional flow field over a trailing-edge serration and implications on broadband noise. Phys. Fluids 28(11), 117101 (2016)

8. Azarpeyvand, M., Gruber, M., Joseph, P.F.: An analytical investigation of trailing edge noise reduction using novel serrations. In: 19th AIAA/CEAS Aeroacoustics Conference (2013)

9. Brandt, A.: Noise and Vibration Analysis: Signal Analysis and Experimental Procedures. 2nd end. Wiley (2011). ISBN:978-0470-74644-8

10. Braslow, A.L., Hicks, R.M., Harris, R.V. Jr.: Use of grit-type boundary-layer transition trips on wind-tunnel models. NASA Technical Note (D-3579) (1966)

11. Chong, T.P., Vathylakis, A.: On the aeroacoustic and flow structures developed on a flat plate with a serrated sawtooth trailing edge. J. Sound Vib. 354, 65-90 (2015)

12. Dassen, T., Parchen, R., Bruggeman, J., Hagg, F.: Results of a wind tunnel study on the reduction of airfoil self-noise by the application of serrated blade trailing edges. Technical report, National Aerospace Laboratory, NLR (1996)

13. Gruber, M.: Airfoil Noise Reduction by Edge Treatments. Ph.D. thesis, University of Southampton (2012)

14. Gruber, M., Joseph, P., Azarpeyvand, M.: An experimental investigation of novel trailing edge geometries on airfoil trailing edge noise reduction. In: 19th AIAA/CEAS Aeroacoustics Conference. AIAA, Berlin, Germany (2013)

15. Gruber, M., Joseph, P., Chong, T.: On the mechanisms of serrated airfoil trailing edge noise reduction. In: 17th AIAA/CEAS Aeroa- 
coustics Conference (32nd AIAA Aeroacoustics Conference), Vol. 2781, pp. 5-8. American Institute of Aeronautics and Astronautics, Portland, Oregon, USA (2011)

16. Howe, M.S.: Noise produced by a sawtooth trailing edge. J. Acoust. Soc. Am. 90(1), 482 (1991)

17. Hurault, J., Gupta, A., Sloth, E., Nielsen, N.C., Borgoltz, A., Ravetta, P.: Aeroacoustic wind tunnel experiment for serration design optimisation and its application to a wind turbine rotor. In: 6th International Meeting on Wind Turbine Noise. Glasgow (2015)

18. Johnson, D.H., Dudgeon, D.E.: Array Signal Processing, Concepts and Techniques. P T R Prentice Hall, Englewood Cliffs (1993). ISBN:9780130485137

19. Jones, L.E., Sandham, N.D., Sandberg, R.D.: Acoustic source identification for transitional airfoil flows using cross correlations. AIAA J. 48(10), 2299-2312 (2010)

20. Lyu, B., Azarpeyvand, M., Sinayoko, S.: Noise prediction for serrated leading-edges. In: 22nd AIAA/CEAS Aeroacoustics Conference (2016)

21. Mathew, J., Singh, A., Madsen, J., León, C.A.: Serration design methodology for wind turbine noise reduction. J. Phys. Conf. Ser. 753, 022019 (2016)

22. Moreau, D.J., Doolan, C.J.: Noise-reduction mechanism of a flatplate serrated trailing edge. AIAA J. 51(13), 2513-2522 (2013)

23. Mueller, T.J.: Aeroacoustic Measurements. Springer Science \& Business Media (2002). ISBN:978-3-642-07514-8

24. Oerlemans, S.: Reduction of wind turbine noise using blade trailing edge devices. In: 22nd AIAA/CEAS Aeroacoustics Conference, pp. 1-18 (2016)

25. Pagani Jr., C.C., Souza, D.S., Medeiros, M.A.F., Medeiros, M.A.F.: Slat noise: aeroacoustic beamforming in closed-section wind tunnel with numerical comparison. AIAA J. 54(7), 2100-2115 (2016)
26. Pröbsting, S., Zamponi, M., Ronconi, S., Guan, Y., Morris, S.C., Scarano, F.: Vortex shedding noise from a beveled trailing edge. Int. J. Aeroacoustics 15(8), 712-733 (2016)

27. Salas, P., Moreau, S.: Noise prediction of a simplified high-lift device. In: 22nd AIAA/CEAS Aeroacoustics Conference. May 30 June 1 2016. Lyon, France., AIAA paper 2016-2962 (2016)

28. Sarradj, E., Herold, G., Sijtsma, P., Merino-Martinez, R., Geyer, T.F., Bahr, C.J., Porteous, R., Moreau, D.J., Doolan, C.J.: A microphone array method benchmarking exercise using synthesized input data. In: 23rd AIAA/CEAS Aeroacoustics Conference. June 5-9 2017. Denver, CO, USA., Abstract submitted for the conference (2017)

29. Sijtsma, P.: Phased array beamforming applied to wind tunnel and fly-over tests. Technical Report NLR-TP-2010-549, National Aerospace Laboratory (NLR), Anthony Fokkerweg 2, 1059 CM Amsterdam, P.O. Box 90502, 1006 BM Amsterdam, The Netherlands (2010)

30. Sijtsma, P.: Analytical benchmark 1. In: 22nd AIAA/CEAS Aeroacoustics Conference. May 30-June 1 2016. Lyon, France., Presentation in Microphone Array Methods Discussion Panel (2016)

31. Tester, B.J.: Engine noise source breakdowns from an improved inverse method (AFINDS) of processing phased array measurements. In: 20th AIAA/CEAS Aeroacoustics Conference. June 16-20 2014. Atlanta GA, USA. AIAA paper 2014-3067 (2014)

32. Yardibi, T., Li, J., Stoica, P., Zawodny, N.S., Cattafesta III, L.N.: A covariance fitting approach for correlated acoustic source mapping. J. Acoust. Soc. Am. 127(5), 2920-2931 (2010) 